\title{
Increased soluble ST2 and IL-4 serum levels are associated with disease severity in patients with membranous nephropathy
}

\author{
ZHIHUI ZHANG ${ }^{1-3^{*}}$, XIAOLEI LIU ${ }^{2 *}$, HAIFENG WANG ${ }^{1,2^{*}}$, ZHIHUI QU ${ }^{4}$, REBECCA CREW $^{5}$, \\ NAN ZHANG ${ }^{1,2}$ and YANFANG JIANG ${ }^{1,2,6}$ \\ ${ }^{1}$ Genetic Diagnosis Center; ${ }^{2}$ Key Laboratory of Zoonoses Research, Ministry of Education, The First Hospital of \\ Jilin University, Changchun, Jilin 130021; ${ }^{3}$ Department of Reproductive Medicine, The Affiliated Hospital of \\ Jining Medical University, Jining, Shandong 272000; ${ }^{4}$ Department of Nephrology, The First Hospital of \\ Jilin University, Changchun, Jilin 130021, P.R. China; ${ }^{5}$ University of Oklahoma Health Sciences Center, \\ Oklahoma, OK 73104, USA; ${ }^{6}$ Jiangsu Co-innovation Center for Prevention and Control of \\ Important Animal Infectious Diseases and Zoonoses, Yangzhou, Jiangsu 225009, P.R. China
}

Received January 5, 2017; Accepted November 13, 2017

DOI: $10.3892 / \mathrm{mmr} .2017 .8130$

\begin{abstract}
The interleukin (IL)-33/suppression of tumorigenicity 2 (ST2) axis regulates Th2 reactivity, and ST2 is the receptor for IL-33. In this study, the roles of IL-33 and soluble ST2 (sST2) in the pathogenesis of membranous nephropathy $(\mathrm{MN})$, and their association with disease severity were evaluated. Serum levels of IL-33 and sST2 in 93 patients, and 34 healthy controls (HCs) were measured by enzyme-linked immunosorbent assays. Clinical characteristics were recorded and the estimated glomerular filtration rates (eGFRs) were computed. In addition, the association between serum IL-33 and sST2 levels, and clinical measurements in patients with $\mathrm{MN}$ was analyzed. No difference in the serum levels of IL-33 was identified between the patients with MN and HCs. However, the serum levels of sST2 were considerably higher in the MN patients compared with in the HCs at every stage. Higher concentrations of serum IL-2, IL-4, IL-10, IL-17A, and IFN- $\gamma$ were measured in the MN patients compared with in the HCs. Serum sST2 concentrations were negatively correlated with IL-4 concentrations in the patients with MN. Furthermore, serum sST2 levels were negatively correlated with the eGFRs and serum calcium levels. Serum sST2 levels, but not IL-33 levels, were positively correlated with the 24-h urine protein and serum phosphorus levels. Following treatment, serum sST2 levels were considerably reduced, whereas serum IL-4
\end{abstract}

Correspondence to: Professor Yanfang Jiang, Genetic Diagnosis Center, The First Hospital of Jilin University, 71 Xinmin Street, Changchun, Jilin 130021, P.R. China

E-mail: yanfangjiang@hotmail.com

*Contributed equally

Key words: interleukin-33, soluble ST2, IL-4, IL-10, membranous nephropathy and IL-10 levels were significantly increased. These data suggest that SST2 and IL-4, but not IL-33, contribute to the pathogenesis of $\mathrm{MN}$.

\section{Introduction}

Membranous nephropathy (MN), the most common cause of adult nephrosis, is an antibody-mediated glomerular illness characterized by subepithelial immune complex deposits, including antigen and complement components $(1,2)$. The clinical symptoms of MN are usually benign or painless; however, 30-40\% of patients develop progressive nephrotic impairments that result in end-stage renal failure within 5-15 years (3). The various types of MN make it difficult to determine the best treatment method for each patient $(4,5)$. MN is an immune complex-mediated nephritogenic immunoreaction. The pathogenesis of MN has not been clarified; therefore, finding biomarkers for the early diagnosis and classification of MN severity is necessary (6).

IL-33 is a member of the IL-1 cytokine family, which includes IL-1a, IL-1b, IL-1 receptor antagonists; in addition, IL-33 and can be produced by epithelial and vascular endothelial cells (7). IL-33 activates various immune cells through binding to the suppression of tumorigenicity 2 (ST2) receptor. This action leads to the production of various molecules and pro-inflammatory cytokines. Once bound to the ST2 acceptor, IL-33 can activate the MyD88 and NF- $\kappa \mathrm{B}$ signaling pathways $(7,8)$. ST2, an IL-33 receptor and IL-1 receptor family member, is located on Th2 cells, mast cells and epithelial cells, but not Th1 cells, and contributes to Th2 activity $(7,9)$. ST2 exists in a transmembrane form (ST2L) and an excretable soluble form of ST2 (sST2). sST2 acts as a decoy receptor by binding to free IL-33 and preventing its signaling via ST2L; however, ST2 plays a role as an intermediary of IL-33 biological activity (10). In fact, the IL-33/ST2 axis plays a vital role in several chronic immune inflammatory disorders, including asthma (7), rheumatoid arthritis (11), and chronic intestinal inflammation (12). Levels of sST2 have been associated with 
the activity and severity of these diseases (13). Nonetheless, there are few studies of whether the IL-33/ST2 axis could be involved in the pathogenesis of membranous nephropathy.

A recent study showed that urinary concentrations of IL-2, IL-4, IL-6, IL-10, IL-17A, IFN- $\gamma$ and TNF- $\alpha$ were considerably higher in MN patients than in healthy controls (HCs) (14). Another study reported no differences in IFN- $\gamma$ and IL-4 mRNA levels in peripheral blood mononuclear cells (PBMCs) in $\mathrm{MN}$ and $\mathrm{HC}$ groups. In contrast, IL-10 mRNA levels in PBMCs were considerably higher in the MN patients than in the HCs (15). Unfortunately, there is little information on variations in serum cytokine levels in MN. Therefore, it is imperative that this area be studied.

To determine the underlying function of IL-33 and sST2 in the pathogenesis of $\mathrm{MN}$, we measured their serum concentrations in MN patients and HCs. Furthermore, we investigated the relation between serum sST2 levels and disease severity. Our data provide new insight into the role of the ST2/IL-33 system in the pathogenesis of MN.

\section{Materials and methods}

Patients. A total of 93 consecutive MN patients from the inpatient service of the First Hospital of Jilin University (Changchun, China) from July 2012 to August 2015 were included in our study. Written informed consent was obtained from all participators. The protocol was established according to the guidelines of the Declaration of Helsinki and was approved by the Human Ethics Committee of the First Hospital of Jilin University (no. 2012-102). Individual patients with MN were diagnosed according to the World Health Organization (WHO) histological classification standards as follows: stage I, most glomerular basement membranes (GBMs) are normal. Only GBM vacuolar degeneration and a small amount of homophilic protein deposited in the epithelium are visible by light microscopy. Electron-dense deposits can be observed by using an electron microscope. Stage II, the GBMs are incrassate. Increased electron density and GBM hyperplasia are visible by electron microscopy. Stage III, the GBMs are visibly incrassate. Electron-dense deposits appear as double-track or chain-like structures. Stage IV, GBM are severely incrassate. The lumen has shrunk, and the renal tubules are sclerous. Electron microscopy show that the electron-dense deposits look like round holes after being dissolved and absorbed. Stage V, the incrassate GBM is gradually restored. Immunopathology shows that immunoglobulin IgG and complement $\mathrm{C} 3$ are deposited along the lateral basolateral membrane with granular and high intensity. Later, this deposition is decreased. Some patients with MN were diagnosed with nephrotic syndrome on the basis of diagnostic standards, including proteinuria (>3.5 g/day), hypoalbuminemia (albumin <30 g/l), dropsy and hyperlipidemia. No patients were treated with hormone medication or other immunosuppressants prior to our research. The levels of ANA, anti-Sm antibody, antineutrophil cytoplasmic antibody (ANCA), viral serology, alexin C3 and $\mathrm{C} 4$, rheumatoid factor, and blood glucose were measured in each patient, and each patient was subjected to an ophthalmological review or an echocardiogram. Exclusion criteria included: i) MN with a fast progression (with a rapid decline in kidney function) and secondary types of MN, such as lupus nephritis, Henoch-Schonlein purpura, and diabetes mellitus; and ii) neoplasia, pregnancy, other autoimmune diseases, kinetic peptic ulcer illness or short-term infections. A total of 34 subjects matched for sex, age and race were included as HCs. Their demographic and clinical features were recorded and analyzed (Table I).

Therapy and follow-up study. After being admitted, individual patients were treated with $1 \mathrm{mg} / \mathrm{kg} /$ day prednisolone (PDN; Tianyao Pharmaceuticals, Tianjin, China) for the first two months. Afterwards, the dose was gradually reduced to $10 \mathrm{mg} / \mathrm{day}$ for maintenance and continued for the next 6 months. The patients were also treated with cyclosporine. Patient follow-ups were conducted for 8-12 weeks. Altogether, 19 patients completed their follow-up, but the other 74 patients did not. Of these 19 patients, 4 were in stage I, 6 were in stage I-II, 8 were in stage II, and 1 was in stage III. After 8-12 weeks of treatment, serum samples were collected during kidney biopsy.

Measurement of serum IL-33 and sST2 levels byenzyme-linked immunosorbent serologic assay (ELISA). Serum IL-33 and sST2 concentrations in MN patients and HCs were measured by human IL-33 (Affymetrix, Santa Clara, CA, USA; eBioscience, San Diego, CA, USA) and sST2 (ImmunoWay, Plano, TX, USA) ELISA kits according to the manufacturer's instructions. Serum IL-33 and sST2 levels in the specimens were calculated using standard curves established from the recombinant IL-33 and sST2 supplied in the kits. The limits of detection for human IL-33 and sST2 were 0.2 and $0.25 \mathrm{pg} / \mathrm{ml}$, respectively.

Cytometric bead array (CBA) analysis of serum cytokines. Serum IFN- $\gamma$, TNF- $\alpha$, IL-2, IL-4, IL-6, IL-10 and IL-17A levels were measured by CBA (BD Biosciences) (16) according to the manufacturer's instructions with minor modifications. Individual serum samples $(25 \mu \mathrm{l})$ were analyzed in duplicate using a FACSCalibur flow cytometry system (BD Biosciences, San Jose, CA, USA) (17), and serum cytokine levels were calculated using CellQuest Pro and CBA software (Becton-Dickinson, San Jose, CA, USA) and a BD FACSAria II system.

Statistical analysis. Data are expressed as the median and range unless specified. Differences between two groups were analyzed by Mann-Whitney U nonparametric tests. Relationships between variables were evaluated by Spearman's rank correlation tests. All statistical analyses were performed using SPSS 16.0 (SPSS, Inc., Chicago, IL, USA) software. Two-sided P-values $<0.05$ were considered to indicate a statistically significant difference.

\section{Results}

Characteristics of $M N$ patients. To determine the potential role of serum IL-33 and sST2 concentrations in the pathogenesis of $\mathrm{MN}, 93$ Chinese patients with newly diagnosed $\mathrm{MN}$ and $34 \mathrm{HCs}$ were recruited for this study. There were no significant differences in the age distribution; serum uric acid, triglyceride, cholesterol or urea nitrogen levels or lymphocyte counts between the groups (Table I). As expected, the MN 


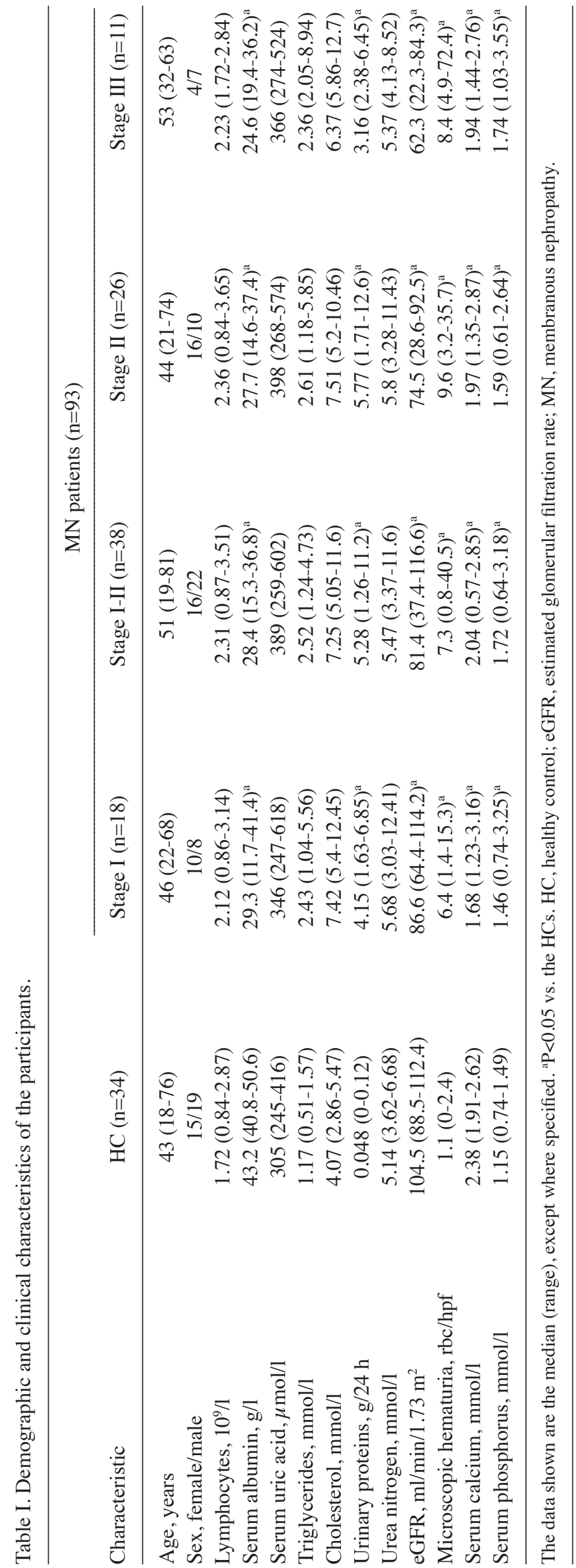



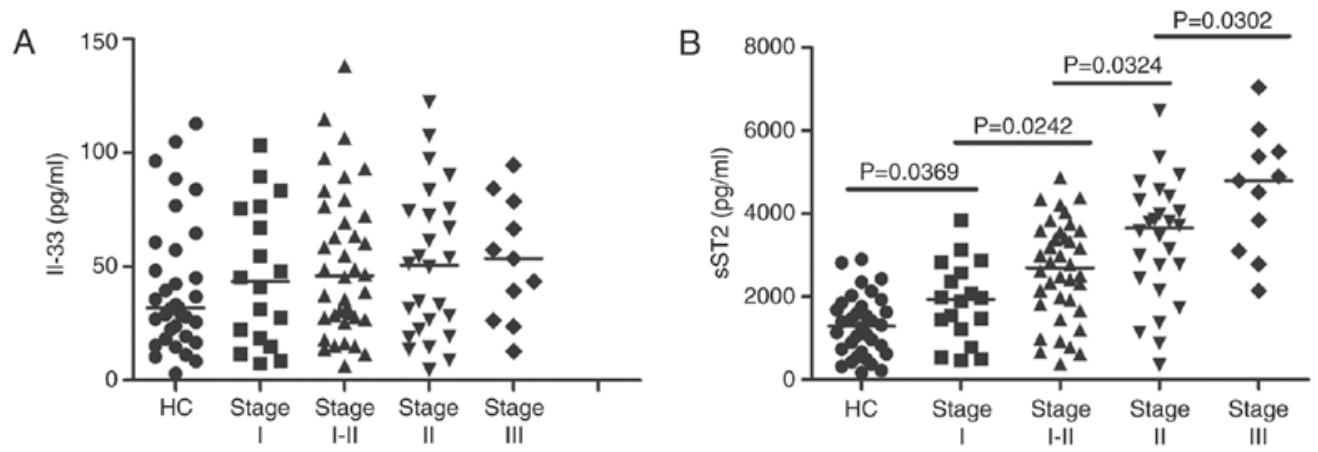

Figure 1. Serum IL-33 and sST2 concentrations. Serum levels of IL-33 and sST2 in individual MN patients and HCs were measured by ELISA. The data are expressed as the average values of individual participants from two independent tests. The horizontal lines represent the median values of different populations. (A) Analyses of serum IL-33 levels revealed no obvious differences between MN patients at different stages and HCs (P>0.05). No significant differences in the serum levels of IL-33 were discovered among MN patients at different stages. (B) Serum sST2 concentrations were significantly higher in MN patients at each stage than in $\mathrm{HCs}(\mathrm{P}<0.05)$. These concentrations were further increased in parallel with the histopathological classification severity of $\mathrm{MN}(\mathrm{r}=0.503$, $\mathrm{P}<0.001)$.
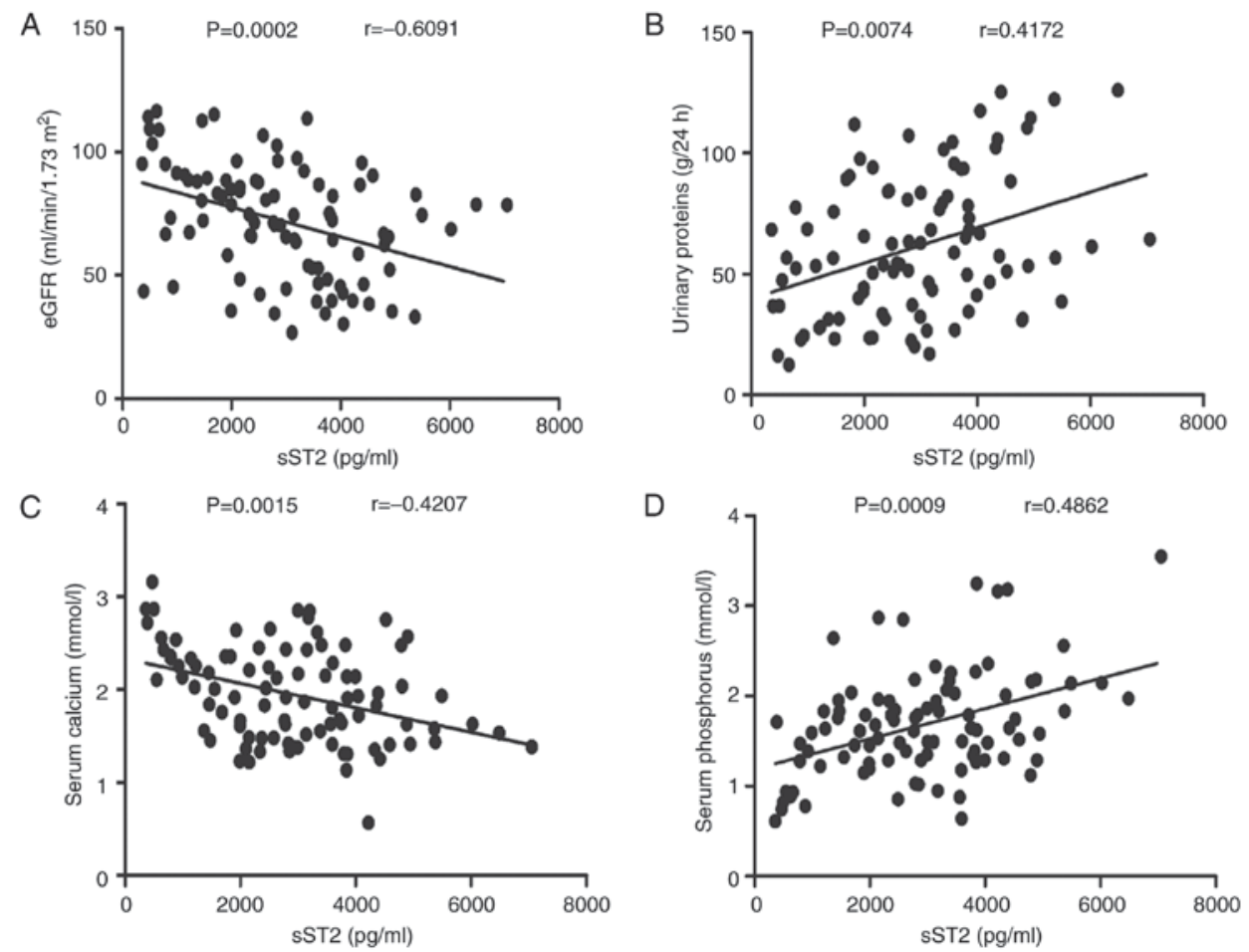

Figure 2. Correlation analyses of serum sST2 levels with clinical parameters in MN patients. The correlations between serum sST2 levels and clinical parameters were determined by using Spearman correlation tests. The data shown are the average values of individual specimens from two independent tests. (A) Serum sST2 levels were negatively correlated with the eGFRs. (B) Serum sST2 levels were positively correlated with the 24-h urinary protein concentrations. (C) Serum sST2 levels were negatively correlated with serum calcium levels. (D) Serum sST2 levels were positively correlated with serum phosphorus levels.

patients had significantly higher 24-h urine protein and serum phosphorus levels than the HCs. However, the estimated glomerular filtration rates (eGFRs) and the serum calcium and albumin levels were considerably lower in the patients than in the HCs. These data suggest that the MN patients had renal dysfunction.

Serum levels of IL-33 and sST2. Serum levels of IL-33 and SST2 were measured in 93 patients and 34 age- and sex-matched HCs. Serum cytokine analyses showed no evident differences in the serum levels of IL-33 between the MN patients at different stages and the HCs (Fig. 1A). In addition, there were no marked differences in the serum levels of IL-33 among MN patients at different stages (data not shown). However, the serum SST2 concentrations were higher in MN patients at all stages than in the HCs $(\mathrm{P}<0.05$; Fig. 1B). Furthermore, the serum sST2 levels were positively correlated with disease severity in the MN patients $(\mathrm{r}=0.503, \mathrm{P}<0.001$; Fig. 1B).

Relationship between the serum levels of SST2 and the clinical features of MN patients. To understand the role of SST2 in the pathogenesis of MN, we evaluated the relationship between the serum sST2 levels and the clinical features of MN patients. In these patients, serum sST2 levels were negatively correlated 
A

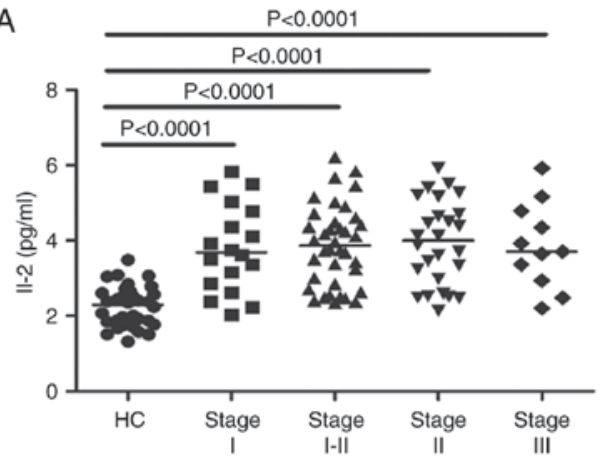

C

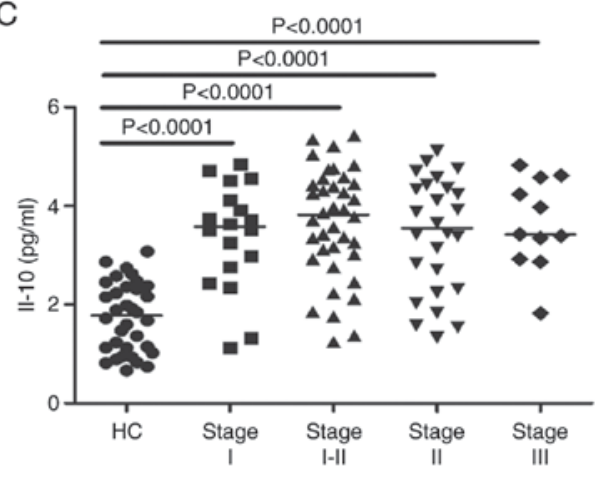

B

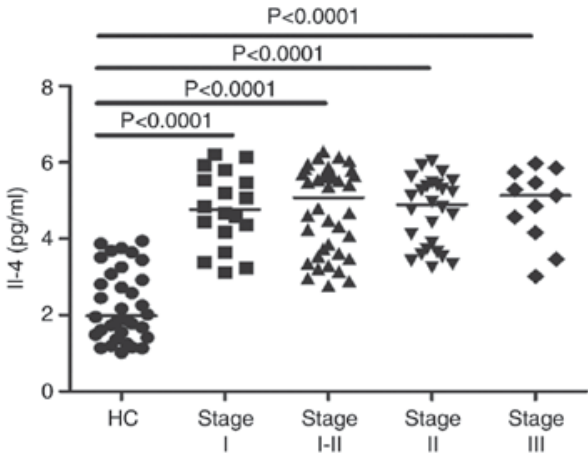

D

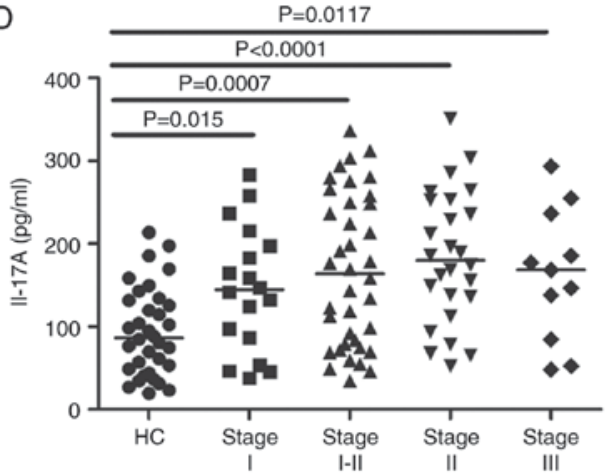

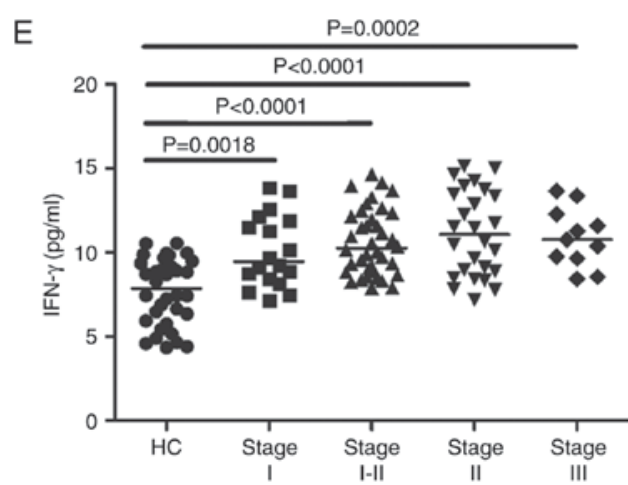

Figure 3. Increased serum concentrations of IL-2, IL-4, IL-10, IL-17A and IFN- $\gamma$ in MN patients before treatment. Serum concentrations of (A) IL-2, (B) IL-4, (C) IL-10, (D) IL-17A and (E) IFN- $\gamma$ were measured via CBA in MN patients and HCs. The data are shown as the average values of individual specimens from two separate tests. The horizontal lines indicate the median values of different clusters.

with the eGFRs ( $\mathrm{r}=-0.6091, \mathrm{P}<0.001$; Fig. $2 \mathrm{~A}$ ) but positively correlated with the 24-h urinary protein $(\mathrm{r}=0.4172, \mathrm{P}=0.0074$; Fig. 2B). Serum sST2 levels were negatively correlated with serum calcium levels ( $\mathrm{r}=-0.4207, \mathrm{P}=0.0015$; Fig. 2C) but positively correlated with serum phosphorus $(r=0.4862$, $\mathrm{P}<0.001$; Fig. 2D) levels. In contrast, the clinical features and IL-2, IL-4, IL-10, IL-17A, IFN- $\gamma$ and IL-33 levels were not correlated (data not shown). Taken together, our research reveals that SST2 may participate in the pathogenesis of MN and play a pivotal role in bone metabolism disorders in $\mathrm{MN}$ patients.

Increased serum concentrations of IL-2, IL-4, IL-10, IL-17A and IFN- $\gamma$ in MN patients before treatment. To determine the potential effects of IL-2, IL-4, IL-10, IL-17A and IFN- $\gamma$ on $\mathrm{MN}$, we measured their serum levels by CBA analysis. The serum levels of IL-2, IL-4, IL-10, IL-17A and IFN- $\gamma$ were considerably higher in the MN patients than in the HCs $(\mathrm{P}<0.05$; Fig. 3A-E). However, the concentrations of those cytokines were not obviously different among MN patients at different stages (data not shown). These data indicate that these cytokines may be involved in the mechanism of MN.

Correlation analyses of the serum levels of SST2 with IL-2, $I L-4, I L-10, I L-17 A, I F N-\gamma$ and $I L-33$ in MN patients. To understand the importance of sST2 in MN, we evaluated the relationship between serum sST2 levels and IL-2, IL-4, IL-10, IL-17A, IFN- $\gamma$ and IL-33 levels in MN patients. Serum sST2 levels were negatively correlated with IL-4 levels ( $r=-0.4337$, $\mathrm{P}<0.001 ;$ Fig. 4A). In contrast, there were no significant correlations between serum sST2 levels and IL-2, IL-10, IL-17A, IFN- $\gamma$ or IL-33 levels in MN patients (Fig. 4B-F).

Correlation analyses of serum sST2 levels with IL-4 levels in MN patients at different stages. As shown in Fig. 4A, serum sST2 levels were negatively correlated with IL-4 levels $(\mathrm{r}=-0.4337, \mathrm{P}=0.0002)$. We next analyzed the correlations between serum sST2 levels and IL-4 levels in MN patients 

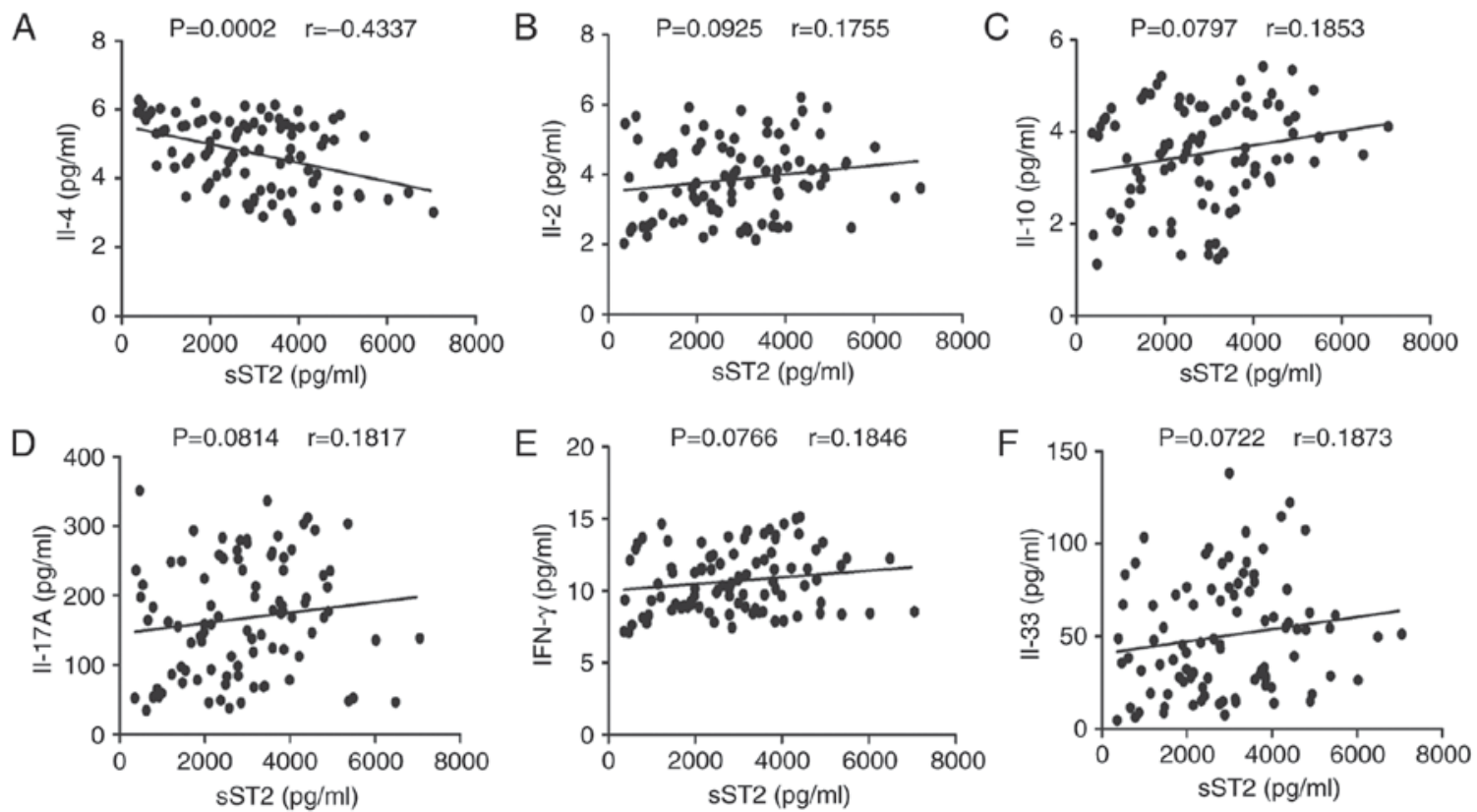

Figure 4. Correlation analyses of serum sST2 levels with IL-2, IL-4, IL-10, IL-17A, IFN- $\gamma$ and IL-33 levels in MN patients. The potential correlations between serum sST2 levels and IL-2, IL-4, IL-10, IL-17A, IFN- $\gamma$ and IL-33 levels were determined by using Spearman correlation tests. The data shown are the averages of individual specimens from two independent tests. (A) Serum sST2 levels were negatively correlated with IL-4 levels. (B-F) No apparent differences were discovered between serum sST2 levels and IL-2, IL-10, IL-17A, IFN- $\gamma$ or IL-33 levels in MN patients.
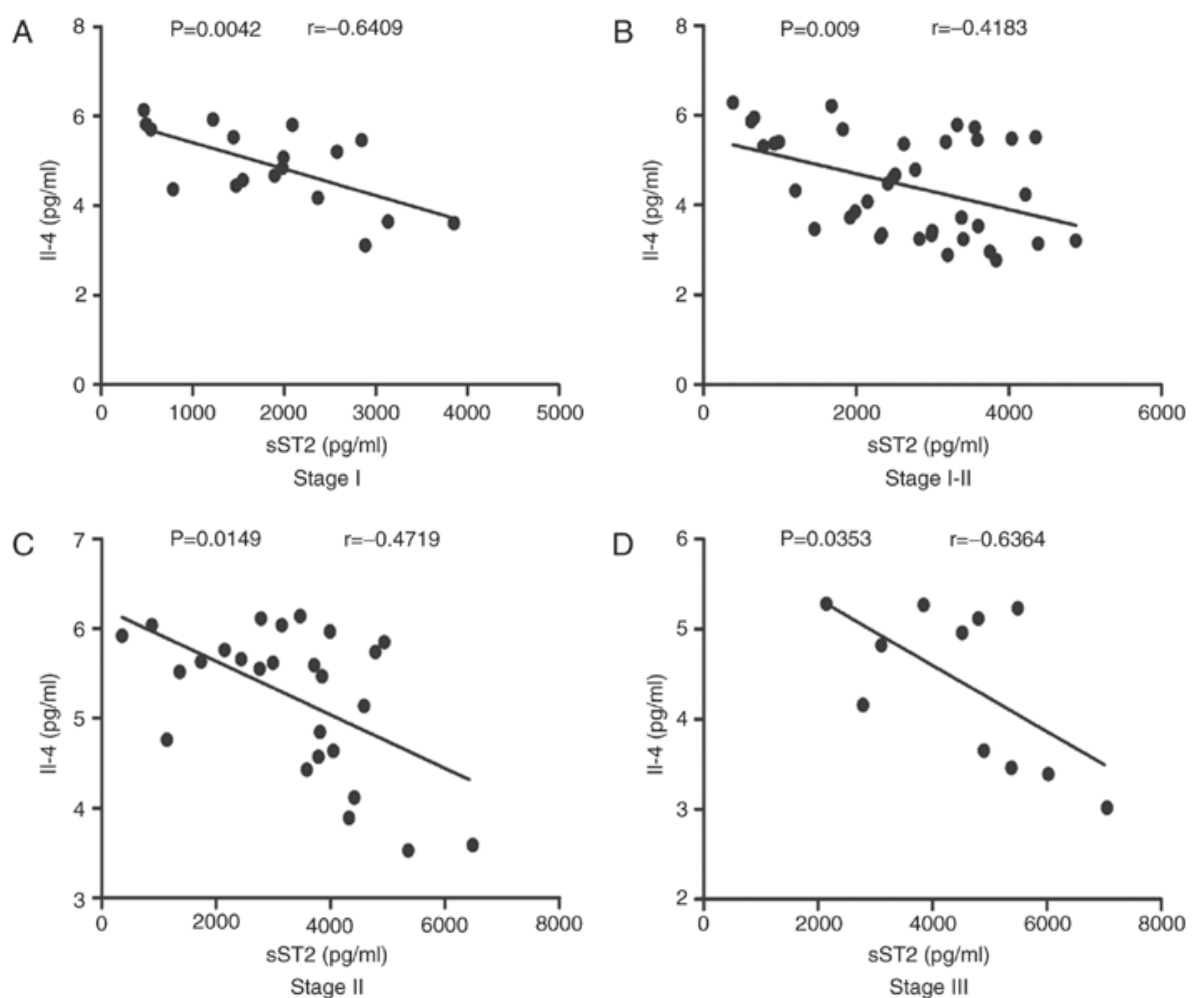

Figure 5. Correlation analyses of serum sST2 levels with IL-4 levels in MN patients at different stages. The relationships between serum sST2 levels and IL-4 levels in MN patients at different stages were determined by using Spearman's correlation tests. The data shown are the averages of individual specimens from two independent tests. (A-D) Serum sST2 levels were negatively correlated with IL-4 levels in stage I, stage I-II, stage II and stage III MN patients.

at different stages. We found that serum sST2 levels were negatively correlated with IL-4 levels in stage I $(\mathrm{r}=-0.6409$, $\mathrm{P}=0.0042$; Fig. 5A), stage I-II ( $\mathrm{r}=-0.4183, \mathrm{P}=0.009$; Fig. 5B), stage II ( $\mathrm{r}=-0.4719, \mathrm{P}=0.0149$; Fig. $5 \mathrm{C})$ and stage III $(\mathrm{r}=-0.6364$, $\mathrm{P}=0.0353$; Fig. 5D) $\mathrm{MN}$ patients.
Clinical parameters and serum cytokine levels in MN patients after treatment. To better understand the role of the IL-33/ST2 axis in MN development, we analyzed the clinical features and serum cytokine concentrations of patients who were followed up for 8-12 weeks. In total, 19 patients had complete records, 
Table II. Effects of treatment on the clinical measurements in the MN patients at follow-up.

\begin{tabular}{lcc}
\hline Characteristic & Before treatment & After treatment \\
\hline Age, years & $48(29-68)$ & $48(29-68)$ \\
Sex, female/male & $10 / 9$ & $10 / 9$ \\
Lymphocytes, $109 / 1$ & $2.34(1.14-3.25)$ & $2.28(1.10-3.11)$ \\
Serum albumin, $\mathrm{g} / \mathrm{l}$ & $28.6(16.8-34.6)$ & $37.2(26.8-46.6)$ \\
Serum uric acid, $\mu \mathrm{mol} / \mathrm{l}$ & $376(253-564)$ & $348(232-523)$ \\
Triglycerides, $\mathrm{mmol} / \mathrm{l}$ & $2.73(1.34-5.42)$ & $2.57(1.18-5.16)$ \\
Cholesterol, mmol/l & $7.22(5.28-11.26)$ & $6.27(4.81-10.13)$ \\
Urinary proteins, $\mathrm{g} / 24 \mathrm{~h}$ & $5.31(1.44-8.26)$ & $3.45(1.78-6.12)^{\mathrm{a}}$ \\
Urea nitrogen, $\mathrm{mmol} / \mathrm{l}$ & $5.74(3.87-10.85)$ & $4.42(2.75-9.18)$ \\
eGFR, ml/min/1.73 m ${ }^{2}$ & $82.36(46.74-102.85)$ & $98.63(62.26-115.28)^{\mathrm{a}}$ \\
Microscopic hematuria, rbc/hpf & $8.65(1.45-67.35)$ & $3.34(0.31-42.23)^{\mathrm{a}}$ \\
Serum calcium, mmol/l & $1.96(1.34-2.43)$ & $2.34(2.05-2.54)^{\mathrm{a}}$ \\
Serum phosphorus, mmol/1 & $1.76(0.93-2.03)$ & $1.17(0.78-1.35)^{\mathrm{a}}$ \\
\hline
\end{tabular}

The data are shown as the median (range). ${ }^{\mathrm{P}}<0.05$ vs. the values before treatment. HC, healthy control; eGFR, estimated glomerular filtration rate; $\mathrm{MN}$, membranous nephropathy.
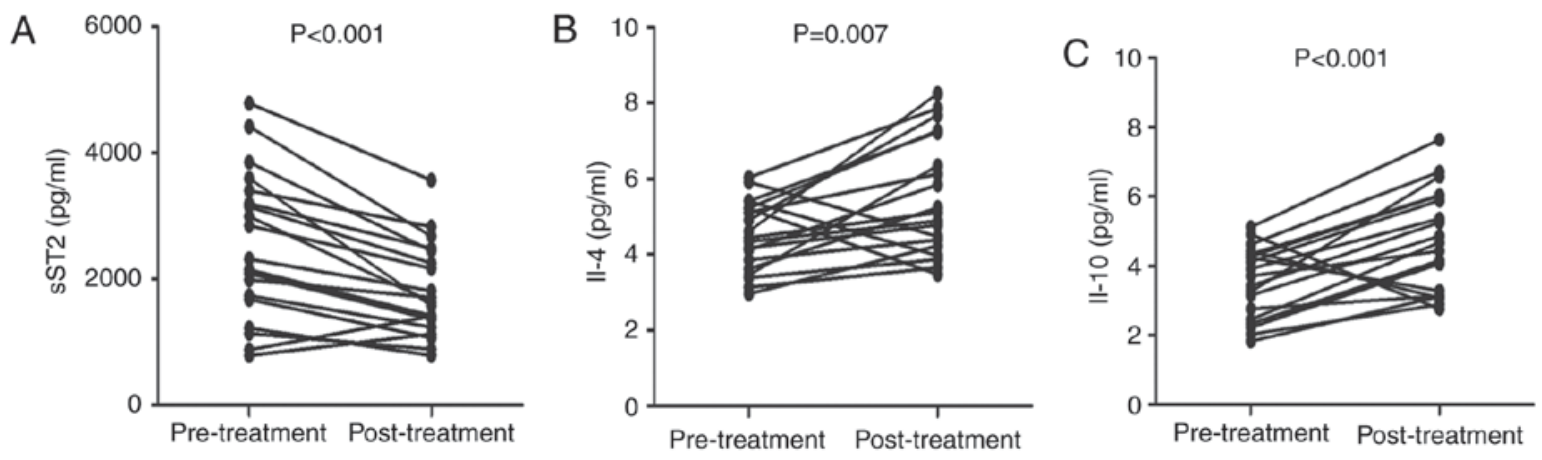

Figure 6. Serum levels of cytokines in MN patients after treatment. Serum concentrations of cytokines in MN patients before and after treatment were analyzed. The data are shown as the mean \% or concentration of individual specimens from two separate tests. (A-C) Serum levels of sST2, IL-4 and IL-10 in individual patients pre and post-treatment.

whereas the other 74 patients failed to complete the follow-up. The 24-h urine protein, microscopic hematuria and serum phosphorus levels were obviously lower in the MN patients than in the HCs, but the eGFRs and serum calcium levels were significantly higher (Table II). In addition, the serum sST2 levels were significantly lower than the pretreatment levels $(\mathrm{P}<0.001$; Fig. 6A). Furthermore, serum IL-4 ( $\mathrm{P}=0.007$; Fig. 6B) and IL-10 ( $\mathrm{P}<0.001$; Fig. 6C) levels were higher after treatment than before treatment. No marked differences were found in the concentrations of other serum cytokines before and after treatment (data not shown). Collectively, MN treatment obviously improved renal function by decreasing the sST2 serum levels and increasing the IL-4 and IL-10 levels in MN patients.

\section{Discussion}

IL-33 is a multipurpose cytokine that participates in several illnesses (13,18-20). Furthermore, IL-33 can activate various immune cells, MAP kinases and NF- $\mathrm{KB}$ signaling pathways and facilitate Th2 reactions through its receptor complex, which comprises ST2 and IL-1RaP (7). When activated by IL-33, structures containing endothelial cells, epithelial cells and fibroblasts produce mediators consistent with the respective cell types. This action exacerbates allergic inflammation. Recently, the IL-33/ST2 axis has been shown to play an important part in several chronic immune inflammatory disorders, including systemic lupus erythematosus (SLE) and rheumatoid arthritis (RA) (21). Serum IL-33 levels are increased in SLE and RA patients and are correlated with the serum erythrocyte sedimentation rate (ESR) and C-reactive protein (CRP) levels; these data indicate that IL-33 may take part in the acute phase of SLE (21). However, the function of the IL-33/ST2 axis in $\mathrm{MN}$ patients remains unclear. Therefore, it is of clinical importance to determine the functions and mechanisms of IL-33 and sST2 in MN patients. This study is the first to measure serum concentrations of IL-33 and sST2 and explore the relationship between the IL-33/ST2 axis and disease severity in MN patients. Recent studies have revealed that sST2 is a negative modulator and competitive decoy receptor for IL-33 $(22,23)$. 
However, in our research, serum IL-33 levels were not increased in MN patients, and IL-33 levels were not correlated with disease severity. These data indicate that IL-33 may not participate in the pathogenesis of $\mathrm{MN}$ or may be inversely downregulated by sST2.

ST2 is selectively expressed by some Th2 cells, but not Th1 cells, and is involved in the Th2 response (9). Importantly, sST2 is a negative modulator of IL-33 and may participate in the pathogenesis of many immune-mediated diseases (22-26). MN is a non-inflammatory organ-specific autoimmune illness that affects the glomerulus and is characterized by the formation of subepithelial immune deposits. When the levels of inflammatory markers increase, kidney function will deteriorate. Our study shows markedly higher sST2 concentrations in MN patients than HCs and indicates that serum sST2 levels are positively correlated with MN disease severity. Following treatment, the serum concentrations of sST2 were obviously lower than those at pretreatment. Furthermore, we discovered that serum sST2 concentrations were positively correlated with the 24-h urinary protein levels but negatively correlated with the eGFRs in MN patients. Our study suggests that increased levels of sST2 may be associated with an increased risk of $\mathrm{MN}$ progression and that serum sST2 levels could be used as a biomarker for assessing $\mathrm{MN}$ disease development and appropriate therapeutic interventions.

Th cells are classified as Th1, Th2, Th17 and Treg cells. Th1 cells produce IFN- $\gamma$ and IL- 2 and facilitate cell-mediated immunity. In contrast, Th2 cells secrete IL-4 and IL-10 and induce antibody production. IL-17 is produced by Th7 cells, mast cells, neutrophils and macrophages. Studies have suggested that IL-17A plays a key role in the mechanisms of host defense, autoimmunity reactions and chronic inflammatory diseases (27). Our study reveals that the serum levels of IL-2, IL-4, IL-10, IL-17A and IFN- $\gamma$ were considerably higher in MN patients than in HCs. Moreover, MN treatment increased the serum levels of IL-4 and IL-10 but did not affect the serum levels of IL-2, IL-17A and IFN- $\gamma$. Our study indicates that pro-inflammatory Th1 and Th17 responses may take part in the pathogenesis of MN and stimulate anti-inflammatory Th2 and Tregs that downregulate pro-inflammatory responses during the pathogenesis of MN.

ST2 expression by Th cells relies on GATA3 signaling, and high levels of sST2 can downregulate the expression of IL-4 but not type 1 cytokines (28). One study reported that the inhibition of IL-33 signaling by sST2 can effectively inhibit the production of IL-4 following allergen exposure in an experimental asthma setting (29). Another study found that sST2 strikingly reduced the levels of IL-4 in mice with airway inflammation compared with those in control mice (30). Furthermore, we found that serum sST2 levels were negatively correlated with IL-4 levels in MN patients. These data indicate that serum sST2 may downregulate IL-4 in MN patients.

Some researchers have found that IL-4 enhances the production of $\mathrm{IgG} 4$ by $\mathrm{B}$ cells in idiopathic $\mathrm{MN}$ in vitro (15). IgG4 is deposited in the glomeruli, which are involved in the pathogenesis of MN (31). Therefore, decreased IL-4 levels may alleviate the course of MN.

Our study shows that the serum calcium levels were lower and that the serum phosphorus levels were higher in
MN patients before treatment than in the HCs. Furthermore, serum sST2 levels were positively correlated with serum phosphorus levels but negatively correlated with serum calcium levels. Following treatment, the serum calcium levels were increased considerably, and the serum phosphorus levels were decreased considerably. Study has indicated that fibroblast growth factor 23 (FGF23) plays a role in reducing parathyroid hormone $(\mathrm{PTH})$ secretion from the parathyroid glands; however, high PTH and FGF23 serum concentrations, in addition to FGF23 resistance in the parathyroid glands, contribute to chronic kidney disease (CKD) (32). FGF23 can act on the nephridium to promote phosphate secretion while inhibiting 25(OH)-vitamin D $1 \alpha-(\mathrm{OH})$ activity and intestinal calcium and phosphorus absorption (33). Importantly, it has been shown that $\mathrm{sST} 2$ may be a regulatory target of PTH that affects bone metabolism (34). One study even showed that CKD patients with elevated PTH levels had higher sST2 levels than HCs (35). MN is the main cause of nephrotic syndrome in adults. Calcium and phosphorus are maintained at relatively constant levels in the blood of healthy subjects, and when phosphorus levels increase, serum calcium levels decrease. In addition, PTH can increase blood calcium levels and reduce blood phosphorus levels. Together, these data suggest that sST2 plays a pivotal role in bone metabolism disorders in MN patients.

In conclusion, our results show that the serum levels of sST2 are considerably higher in MN patients than in HCs. These data suggest that SST2 plays a critical role in the pathogenesis of $\mathrm{MN}$ and can be used as a biomarker for assessing illness seriousness. We admit that our research is limited by its small number of patients and single time-point data for some patients; other limitations include the limited amount of information regarding renal system damage and the lack of data regarding sST2 levels after treatment. Although further study is required to more clearly define the role and mechanism of SST2 in the pathogenesis of MN, our novel research provides new data for understanding this disease.

\section{References}

1. Glassock RJ: The pathogenesis of idiopathic membranous nephropathy: A 50-year odyssey. Am J Kidney Dis 56: 157-167, 2010.

2. Ronco P and Debiec H: Pathogenesis of membranous nephropathy: Recent advances and future challenges. Nat Rev Nephrol 8: 203-213, 2012.

3. Cattran D: Management of membranous nephropathy: When and what for treatment. J Am Soc Nephrol 16: 1188-1194, 2005.

4. Cattran DC: Idiopathic membranous glomerulonephritis. Kidney Int 59: 1983-1994, 2001

5. Honkanen E, Törnroth T and Grönhagen-Riska C: Natural history, clinical course and morphological evolution of membranous nephropathy. Nephrol Dial Transplant 7 (Suppl 1): S35-S41, 1992.

6. Glassock RJ: The treatment of idiopathic membranous nephropathy: A dilemma or a conundrum? Am J Kidney Dis 44: 562-566, 2004.

7. Schmitz J, Owyang A, Oldham E, Song Y, Murphy E, McClanahan TK, Zurawski G, Moshrefi M, Qin J, Li X, et al: IL-33, an interleukin-1-like cytokine that signals via the IL-1 receptorrelated protein ST2 and induces T helper type 2-associated cytokines. Immunity 23: 479-490, 2005.

8. Alves-Filho JC, Sônego F, Souto FO, Freitas A, Verri WA Jr, Auxiliadora-Martins M, Basile-Filho A, McKenzie AN, Xu D, Cunha FQ and Liew FY: Interleukin-33 attenuates sepsis by enhancing neutrophil influx to the site of infection. Nat Med 16: 708-712, 2010. 
9. Löhning M, Stroehmann A, Coyle AJ, Grogan JL, Lin S, Gutierrez-Ramos JC, Levinson D, Radbruch A and Kamradt T: T1/ST2 is preferentially expressed on murine Th2 cells, independent of interleukin 4, interleukin 5, and interleukin 10, and important for Th2 effector function. Proc Natl Acad Sci USA 95: 6930-6935, 1998.

10. Sanada S, Hakuno D, Higgins LJ, Schreiter ER, McKenzie AN and Lee RT: IL-33 and ST2 comprise a critical biomechanically induced and cardioprotective signaling system. J Clin Invest 117: 1538-1549, 2007.

11. Palmer G, Talabot-Ayer D, Lamacchia C, Toy D, Seemayer CA, Viatte S, Finckh A, Smith DE and Gabay C: Inhibition of interleukin-33 signaling attenuates the severity of experimental arthritis. Arthritis Rheum 60: 738-749, 2009.

12. Pastorelli L, De Salvo C, Cominelli MA, Vecchi M and Pizarro TT: Novel cytokine signaling pathways in inflammatory bowel disease: Insight into the dichotomous functions of IL-33 during chronic intestinal inflammation. Therap Adv Gastroenterol 4: 311-323, 2011

13. Mok MY, Huang FP, Ip WK, Lo Y, Wong FY, Chan EY, Lam KF and Xu D: Serum levels of IL-33 and soluble ST2 and their association with disease activity in systemic lupus erythematosus. Rheumatology (Oxford) 49: 520-527, 2010.

14. Kalavrizioti D, Gerolymos M, Rodi M, Kalliakmani P, Provatopoulou S, Eleftheriadis T, Mouzaki A and Goumenos DS: $\mathrm{T}$ helper (Th)-cytokines in the urine of patients with primary glomerulonephritis treated with immunosuppressive drugs: Can they predict outcome? Cytokine 76: 260-269, 2015.

15. Kuroki A, Iyoda M, Shibata T and Sugisaki T: Th2 cytokines increase and stimulate B cells to produce IgG4 in idiopathic membranous nephropathy. Kidney Int 68: 302-310, 2005.

16. Morgan E, Varro R, Sepulveda H, Ember JA, Apgar J, Wilson J, Lowe L, Chen R, Shivraj L, Agadir A, et al: Cytometric bead array: A multiplexed assay platform with applications in various areas of biology. Clin Immunol 110: 252-266, 2004.

17. Tárnok A, Hambsch J, Chen R and Varro R: Cytometric bead array to measure six cytokines in twenty-five microliters of serum. Clin Chem 49: 1000-1002, 2003.

18. Barksby HE, Lea SR, Preshaw PM and Taylor JJ: The expanding family of interleukin-1 cytokines and their role in destructive inflammatory disorders. Clin Exp Immunol 149: 217-225, 2007.

19. Liew FY, Pitman NI and McInnes IB: Disease-associated functions of IL-33: The new kid in the IL-1 family. Nat Rev Immunol 10: 103-110, 2010.

20. Wang J, Cai Y, Ji H, Feng J, Ayana DA, Niu J and Jiang Y: Serum IL-33 levels are associated with liver damage in patients with chronic hepatitis B. J Interferon Cytokine Res 32: 248-253, 2012.

21. Yang Z, Liang Y, Xi W, Li C and Zhong R: Association of increased serum IL-33 levels with clinical and laboratory characteristics of systemic lupus erythematosus in Chinese population. Clin Exp Med 11: 75-80, 2011

22. Hayakawa H, Hayakawa M, Kume A and Tominaga S: Soluble ST2 blocks interleukin-33 signaling in allergic airway inflammation. J Biol Chem 282: 26369-26380, 2007.
23. Dinarello CA: An IL-1 family member requires caspase-1 processing and signals through the ST2 receptor. Immunity 23 : 461-462, 2005.

24. Brunner M, Krenn C, Roth G, Moser B, Dworschak M, Jensen-Jarolim E, Spittler A, Sautner T, Bonaros N, Wolner E, et al: Increased levels of soluble ST2 protein and IgG1 production in patients with sepsis and trauma. Intensive Care Med 30: 1468-1473, 2004

25. Shimpo M, Morrow DA, Weinberg EO, Sabatine MS Murphy SA, Antman EM and Lee RT: Serum levels of the interleukin-1 receptor family member ST2 predict mortality and clinical outcome in acute myocardial infarction. Circulation 109: 2186-2190, 2004

26. Mueller T, Dieplinger B, Gegenhuber A, Poelz W, Pacher R and Haltmayer M: Increased plasma concentrations of soluble ST2 are predictive for 1-year mortality in patients with acute destabilized heart failure. Clin Chem 54: 752-756, 2008.

27. Rodrigues-Díez R, Aroeira LS, Orejudo M, Bajo MA, Heffernan JJ, Rodrigues-Díez RR, Rayego-Mateos S, Ortiz A, Gonzalez-Mateo G, López-Cabrera M, et al: IL-17A is a novel player in dialysis-induced peritoneal damage. Kindey Int 86: 303-315, 2014.

28. Griesenauer B and Paczesny S: The ST2/IL-33 axis in immune cells during inflammatory diseases. Front Immunol 8: 475, 2017.

29. Hayakawa H, Hayakawa M, Kume A and Tominaga S: Soluble ST2 blocks IL-33 signaling in allergic airway inflammation. J Biol Chem 282: 26369-26380, 2007.

30. Yin H, Li XY, Liu T, Yuan BH, Zhang BB, Hu SL, Gu HB, Jin XB and Zhu JY: Adenovirus-mediated delivery of soluble ST2 attenuates ovalbumin-induced allergic asthma in mice. Clin Exp Immunol 170: 1-9, 2012.

31. Prunotto M, Carnevali ML, Candiano G, Murtas C, Bruschi M, Corradini E, Trivelli A, Magnasco A, Petretto A, Santucci L, et al: Autoimmunity in membranous nephropathy targets aldose reductase and SOD2. J Am Soc Nephrol 21: 507-519, 2010.

32. Lavi-Moshayoff V, Wasserman G, Meir T, Silver J and Naveh-Many T: PTH increases FGF23 gene expression and mediates the high-FGF23 levels of experimental kidney failure: A bone parathyroid feedback loop. Am J Physiol Renal Physiol 299: F882-F889, 2010.

33. Shimada T, Hasegawa H, Yamazaki Y, Muto T, Hino R, Takeuchi Y, Fujita T, Nakahara K, Fukumoto S and Yamashita T: FGF-23 is a potent regulator of vitamin D metabolism and phosphate homeostasis. J Bone Miner Res 19: 429-435, 2004.

34. Torres VE: Treatment strategies and clinical trial design in ADPKD. Adv Chronic Kidney Dis 17: 190-204, 2010.

35. Bao YS, Na SP, Zhang P, Jia XB, Liu RC, Yu CY, Mu SH and Xie RJ: Characterization of interleukin-33 and Soluble ST2 in serum and their association with disease severity in patients with chronic kidney disease. J Clin Immunol 32: 587-594, 2012. 